\section{PREVENCIÓN DEL RIESGO A ESCALA LOCAL Y SENSIBLE AL GÉNERO: EXPERIENCIA Y APRENDIZAJES DESDE ACAPULCO, MÉXICO}

Celia Ramírez ${ }^{1}$ y Héctor Becerril 2*

\section{RESUMEN}

Este trabajo plantea una estrategia de prevención del riesgo a escala local y sensible al generó, buscando contribuir a delinear formas de concretar procesos inclusivos de prevención y reducción de riesgos. La estrategia se basa en una investigación-acción participativa realizada entre 2018 y 2019 con mujeres de la Colonia Palma Sola FOVISSSTE, en la periferia de Acapulco de Juárez, México. Metodológicamente, el trabajo incluyó tres ejes complementarios de actuación: involucramiento y sensibilización, diagnóstico y planeación, y vinculación y acción. Este trabajo enfatiza la importancia de generar estrategias sostenidas por procesos de involucramiento que busquen entrelazar gestión del riesgo y desarrollo local, y procesos de vinculación que permitan la creación de alianzas con actores gubernamentales y no gubernamentales. También se subrayan tres desafíos clave entorno al desarrollo de procesos de prevención a escala local sensibles al género: romper con los estereotipos y roles de género, diseñar procesos y actividades a la medida de los intereses, necesidades y capacidades de las mujeres, y fomentar el empoderamiento de las mujeres, buscado en particular mejorar su autoestima y expandir sus capacidades para relacionarse y crear alianzas multisectoriales.

\section{PALABRAS CLAVES}

Gestión del riesgo, Desarrollo local, Género, Empoderamiento, México

\section{RISK PREVENTION AT LOCAL SCALE AND GENDER-SENSITIVE: EXPERIENCE AND LEARNING FROM ACAPULCO, MEXICO}

\section{ABSTRACT}

This work proposes a risk prevention strategy at a local scale and gender sensitive, aiming to contribute to delineate ways to materialize inclusive processes of prevention and risk reduction. The strategy is based on a participatory 'action research' carried out between 2018 and 2019 with women from Colonia Palma Sola FOVISSSTE, on the outskirts of Acapulco de Juárez, Mexico. Methodologically, the work included three complementary lines of action: involvement and awareness, diagnosis and planning, and linkage and action. This work emphasizes the importance of generating strategies sustained by involvement processes that seek to link risk management and local development, and linkage processes that allow the creation of alliances with governmental and non-governmental actors. This work also highlights three key challenges around developing gender-sensitive local management processes: breaking gender stereotypes and roles, designing processes and activities tailored to the interests, needs and capacities of women, and promoting the empowerment of women, seeking in particular to improve their self-esteem and expand their capacities to relate and create multisectoral alliances.

\section{KEYWORDS}

Risk management, Local development, Gender, Empowerment, Mexico
1. Instituto Integral para la Gestión Social del Riesgo de Desastre y Cambio Climático A.C., Acapulco, México.

2. Consejo Nacional de Ciencia y Tecnología CONACYT, Universidad Autónoma de Guerrero, Acapulco, México.

*Autor de correspondencia: hbecerrilmi@uagro.mx

Identificador:

http://revistareder.com/ handle-0719-8477-2020-083

\section{RECIBIDO}

10 de noviembre de 2020

\section{ACEPTADO}

2 de diciembre de 2020

PUBLICADO

1 de enero de 2021

\section{Formato cita}

Recomendada (APA): Ramírez, C. \& Becerril, H. (2021). Prevención del Riesgo a Escala Local y Sensible al Género: Experiencia y Aprendizajes desde Acapulco, México. Revista de Estudios Latinoamericanos sobre Reducción del Riesgo de Desastres REDER, 5(1), 1327. http://revistareder.com/ handle-0719-8477-2020-083

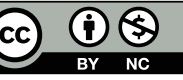

Todos los artículos publicados en REDER siguen una política de Acceso Abierto y se respaldan en una Licencia CreativeCommons Atribución-NoComercial 4.0 Internacional.

Revista de Estudios

Latinoamericanos sobre Reducción del Riesgo de Desastres (REDER)

Diseño: Lupe Bezzina 


\section{INTRODUCCIÓN}

Los riesgos de desastres asociados a fenómenos naturales representan uno de los principales desafíos de América Latina y el Caribe (ALC): entre 2000 y 2019 se registraron 1,205 desastres que afectaron a 152 millones de personas, convirtiéndose en la segunda región a nivel global más expuesta de acuerdo con la Oficina de Naciones Unidas para la Coordinación de Asuntos Humanitarios -OCHA por sus siglas en inglés- (OCHA, 2020). Este desafío se espera que sea exacerbado por el cambio climático puesto que la región es especialmente vulnerable a sus impactos potenciales (CEPAL, 2018), dentro de los que destacan, un incremento de la probabilidad de déficit de precipitaciones y sequias en ciertas regiones, y el aumento de la probabilidad de fuertes precipitaciones en otras. Asimismo se pronostica un aumento de la temperatura media en los océanos y la tierra, y extremos calientes en la mayoría de las regiones habitadas (IPCC, 2018).

En la región, la gestión de dichos riesgos se ha hecho principalmente a partir de enfoques reactivos y correctivos, con escasa articulación con la planeación territorial, y limitada participación de la sociedad civil, en particular de las mujeres. Frente a esto, existe consenso sobre la importancia de transitar hacia procesos inclusivos de prevención y reducción de riesgos a escala local, atendiendo las causas subyacentes del riesgo tales como las desiguales de género, e incentivando la participación de las personas, y en particular la participación y liderazgo de las mujeres (Castro, 2017; PNUD, 2007; UNDRR, 2015b). Buscando contribuir a este fin, este trabajo plantea una estrategia de prevención del riesgo a escala local y sensible al generó desarrollada a través de un proceso de investigación-acción participativa realizado entre 2018 y 2019 con mujeres de la Colonia Palma Sola FOVISSSTE, en la periferia de Acapulco (ver Figura 1), marcada por condiciones de precariedad y vulnerabilidad ante desastre. El trabajo este guiado por la siguiente pregunta: cómo concretar y sostener procesos de prevención del riesgo a escala local que sean sensibles al género.

Este trabajo subraya la relevancia de desarrollar estrategias de prevención sostenidas, por un lado, por procesos de involucramiento que entrelacen gestión del riesgo y desarrollo local, atendiendo las desigualdades de género; y por el otro, por procesos de vinculación que permitan a las mujeres crear alianzas con actores gubernamentales y no gubernamentales. Este trabajo también subraya los siguientes desafíos en torno al desarrollo de una gestión del riesgo con enfoque de género: romper con los estereotipos y roles de género, diseñar procesos y actividades a la medida de los intereses, necesidades y capacidades de las mujeres, y fomentar el empoderamiento de las mujeres, buscado en particular mejorar su autoestima y expandir sus capacidades para relacionarse y crear alianzas multisectoriales. El articulo comporta seis apartados. En el primero se presenta el marco referencial mientras que el segundo da cuenta de la metodología empleada. Posteriormente, el tercer apartado presenta el caso de estudio, y el cuarto los resultados. El quinto apartado discute los resultados y el ultimo presenta las conclusiones.

\section{MARCO REFERENCIAL}

Este trabajo se basa en el entendimiento del riesgo como una "construcción social, dinámica y cambiante" que resulta de relación entre amenazas y vulnerabilidades, y la cual es situada territorial y socialmente. Específicamente, se entiende que el riesgo "se concreta, se mide, se enfrenta y se sufre" de manera clara a nivel micro territorial y social, pues es ahí donde pasa "de una condición latente en una condición de perdida, crisis o desastre" (Lavell, 2008, p. 5). Por ello, se concibe la gestión del riesgo como la concreción de acciones que buscan reducir los riesgos de desastres tales como la prevención, mitigación, y preparación (UNDRR, 2015a), transformando las "amenazas y los factores de vulnerabilidad en oportunidades de cambio positivo" (Wilches-Chaux, 1998, p. 75). Más aun, este trabajo concibe que la gestión del riesgo implica considerar y atender las causas subyacentes del riesgo, ya que las pérdidas humanas y materiales de los desastres están relacionadas tanto con la magnitud de los eventos como con las desigualdades sociales y desequilibrios ambientales de los territorios (Castro, 2017). De ahí que se requiere incentivar la prevención y adaptación, a través de la reducción de vulnerabilidades, la disminución de la exposición de las poblaciones y sus bienes, y la preparación de las personas; en suma "estrategias de desarrollo basadas en procesos de transformación que disminuyan la vulnerabilidad social y aumente las capacidades de gestión y adaptación" (Castro, 2017, p. 2).

Esta conceptualización está en línea con el Marco de Sendai, el cual busca prevenir nuevos riesgos, reducir los riesgos existentes y reforzar la resiliencia, buscando consolidar tanto la 
gestión del riesgo como los procesos de desarrollo. Todo ello, a través de acciones integrales y multisectoriales orientadas a las personas sus bienes y medios de vida, y desarrolladas en colaboración con toda la sociedad (UNDRR, 2015b). Es importante señalar que en los últimos años se ha buscado articular de manera clara y explicita la reducción del riesgo, el cambio climático y el desarrollo sostenible, enfatizando la importancia de la gestión del riesgo y la acción climática para lograr la Agenda 2030 (IPCC, 2018; UNDRR, 2019; Zhenmin \& Espinosa, 2019). Por otro lado, este trabajo se alinea con el Marco de Sendai sobre la necesidad de generar procesos de "empoderamiento y una participación inclusiva, accesible y no discriminatoria, prestando especial atención a las personas afectadas desproporcionadamente por los desastres, en particular las más pobres"; e incitar la adopción de una perspectiva de género y la promoción del liderazgo de las mujeres (UNISDR, 2015, parrafo 19).

El empoderamiento se puede entender como un proceso gracias al cual las personas adquieren control y poder sobre sus vidas a través de "la toma de conciencia, la construcción de la autoconfianza, la ampliación de las opciones y oportunidades y el creciente acceso y control de los recursos" (Aguilar, Castañeda, \& Salazar, 2000, p. 41). Por ello, el empoderamiento no es algo que se otorga, si no que las personas logran. Específicamente, este trabajo entiende el empoderamiento de las mujeres como un proceso de cambio durante el cual estas van ganando poder a fin de "transformar la ideología, las estructuras e instituciones que refuerzan y perpetúan la discriminación de género", mediante "la capacitación, la participación, la organización y el ejercicio de la ciudadanía" (Aguilar, Castañeda, \& Salazar, 2000, p. 41).

Por su parte, el enfoque de género implica visibilizar, entender y atender las diferencias de poder y condiciones entre hombres y mujeres basadas en género. De acuerdo con el Programa de las Naciones Unidas para el Desarrollo (PNUD), existen múltiples factores de género relacionados con el aumento de la vulnerabilidad de las mujeres en torno a la gestión del riesgo. Entre dichos factores destacan los bajos ingresos económicos y condiciones de pobreza, el analfabetismo y las desigualdades con relación a oportunidades educativas, y un restringido acceso a recursos financieros y tecnológicos. También destacan la precaria participación en el liderazgo de las organizaciones vinculadas a la gestión del riesgo, el exiguo acceso a la toma de decisión dentro y fuera de los hogares, y dificultades para participar en capacitaciones. Por último, otros factores de género relacionados con la vulnerabilidad de las mujeres son el riesgo de violencia dentro y fuera de los hogares, y restricciones para realizar actividades y circular fuera del espacio doméstico (PNUD, 2007). De acuerdo con Boyano (2013), los factores de género se ven exacerbados por la escasa integración de una perspectiva de género en las normas y procedimientos relacionados con la gestión del riesgo. Por su parte, Arana (2017, p. 3) señala que la vulnerabilidad de las mujeres ante la crisis climática incrementa tanto por factores biológicos como socioculturales relacionados con el género: "generalmente las mujeres tienen limitado acceso a los activos que pueden mejorar su capacidad de adaptación al cambio climático: escolaridad, acceso a la tierra, agua y créditos, participación en la toma de decisiones, capacitación y tecnología". Asimismo, Arana (2017) enfatiza que las niñas y las mujeres poseen "fortalezas y potencialidades" para afrontar el cambio climático: entre otros tienen la capacidad de generar redes de distribución y servicios, incrementando el acceso a energías renovables y reduciendo costos; y tiene un rol central en la transición energética al ser las principales administradoras de energía en los hogares. No obstante, estas fortalezas y potencialidades son ignoradas.

Por lo anterior, una gestión del riesgo sensible al género es relevante ya que permite discernir como las vulnerabilidades y capacidades son determinadas por las identidades de hombres y mujeres, generando condiciones de riesgos diferenciados; y desarrollar iniciativas que ayuden a lograr una mayor equidad y autonomía de las mujeres (PNUD, 2007).

\section{MÉTODOS}

Este trabajo se basa en una investigación realizada entre 2018 y 2019 desde un enfoque de investigación-acción participativa (IAP) con un grupo de aproximadamente 30 mujeres de la Colonia Palma Sola FOVISSSTE localizada en la periferia de Acapulco de Juárez y marcada por condicione de precariedad y vulnerabilidad ante los riesgos de desastre. La IAP es un enfoque de investigación que está a favor de una práctica de generación de conocimiento que sea "explícitamente política, socialmente inmersa y democrática” (Brydon-Miller, Greenwood, \& Maguire, 2003, p.13), la cual contribuya a transformar los patrones sociales económicos y políticos no democráticos e injustos. 
Cabe resaltar que un elemento clave de la IAP es el reconocimiento del conocimiento de las personas y de sus capacidades para enfrentar los problemas que los desafían, por lo cual su involucramiento es clave en el proceso de investigación (Brydon-Miller, Greenwood, \& Maguire, 2003).

El desarrollo de la investigación en Palma Sola se basó por un lado en las fases generales de la IAP: la preparación de un diagnóstico, la elaboración de planes de acción, y la implementación de estos; todo ello, a través de una constate reflexión que permitió "redimensionar, reorientar o replantear nuevas acciones" (Colmenares, 2012, p.107). Por el otro, la investigación se basó en la ruta metodológica para atender el cambio climático elaborada por la asociación Espacio de Encuentro de las Culturas Originarias (EECO, 2016), la cual consta de 5 etapas: sensibilización, diagnostico, identificación de acciones y proyectos y sistema de monitoreo participativo.

En específico, la estrategia en Palma Sola comportó tres ejes de actuación (ver Tabla 1). El primero se centró en el involucramiento y la sensibilización. Por un lado, el implicarse en la colonia, e implicar a las mujeres de la colonia en el proyecto, se fomentó a través la generación de confianza, y la coproducción de conocimiento y acciones a lo largo de toda la intervención, considerando tanto las fortalezas como aspiraciones y necesidades del grupo de mujeres. Por otro lado, para la sensibilización sobre los riesgos de desastres y la crisis climática se usaron diferentes técnicas didácticas, considerando el perfil de las participantes. El segundo eje de actuación se enfocó en la elaboración de un diagnóstico de riesgos participativo y la planeación de acciones para enfrentarlos; esto a través de discusiones grupales, entrevistas semiestructuradas, observación participante, consulta de datos oficiales, trabajo de escritorio y un taller de diagnóstico y planeación participativo. El tercer eje se orientó a la vinculación e implementación del plan de acción. La vinculación consistió en el desarrollo de relaciones y creación de alianzas con actores e instituciones gubernamentales y no gubernamentales, mientras que la implementación consistió en la concreción de las acciones acordadas por el grupo de mujeres.

Además, se buscó desarrollar capacidades y fomentar el empoderamiento de las mujeres de manera transversal a lo largo de la implementación de los ejes antes mencionados. Es importante mencionar que la concreción de los diferentes ejes no se dio de manera linear sino de forma paralela y complementar.

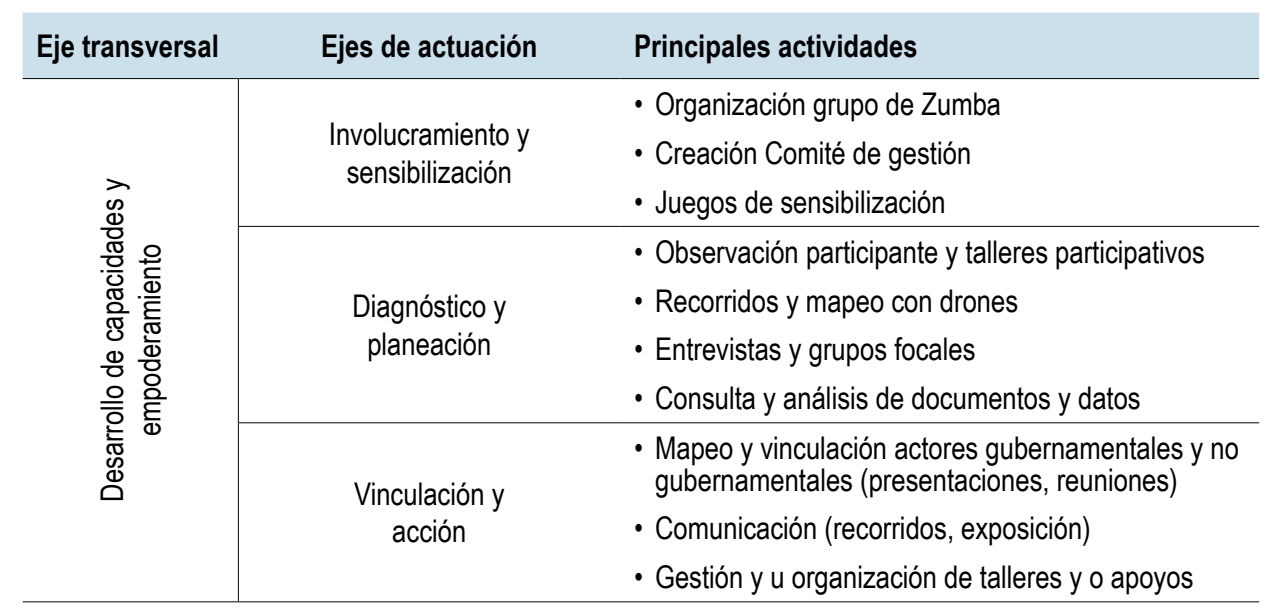

Tabla 1. Estrategia de prevención de riesgos: ejes de actuación y principales actividades Fuente: Autores, 2021.

\section{CASO DE ESTUDIO}

La Colonia Palma Sola Sector FOVISSSTE se localiza en el sector urbano del municipio de Acapulco de Juárez, en estado de Guerrero. Específicamente, se localiza en una ladera del parque El Veladero en el área del Anfiteatro que conforma la bahía de Acapulco (ver Figura 1). La ZMA y en particular Acapulco tuvo un crecimiento poblacional urbano importante durante el siglo XX, pasando de 34,458 habitantes en 1930 a 777,240 en 2010 (Instituto Nacional de Estadística y Geografía [INEGI], s.f.). Este crecimiento fue incentivado por el desarrollo de Acapulco como centro turístico nacional e internacional. Actualmente se estima que la ZMA cuenta con una población de 887,005 (INEGI, 2016) y constituye un clúster urbano intermedio, por su tamaño y rol mediador entre asentamientos urbanos y rurales en el estado y el país (Becerril, Rodríguez \& Yáñez, 2020). 




Figura 1. Localización Colonia Palma Sola FOVISSSTE

Fuente: Elaborado por Laura Guzmán con datos de INEGI (s.f)

De acuerdo con datos de El Consejo Nacional de Evaluación de la Política de Desarrollo Social (CONEVAL, s.f.a) el grado de rezago social en Acapulco es Bajo. No obstante, dentro del municipio y la ZMA existen colonias de alta marginación. Esta situación está relacionada con un desarrollo desigual entre las partes turísticas y las colonias en la periferia urbana marcada por niveles de pobreza alto, viviendas de baja calidad y servicios públicos deficientes o inexistentes. Una de esta colonia es Palma Sola Sector FOVISSSTE que cuenta con una población de aproximadamente 2,547 personas, de las cuales 1,330 son mujeres, equivalente al $52 \%$. De acuerdo con Sistema de Información Social y Georreferenciada (SISGE) la colonia es considerada como una área de alto rezago social (Secretaria Bienestar, s.f.). Entre otros, de acuerdo con el Censo de población de 2010 en la colonia viven 911 personas (36\%) que son no derechohabiente a servicios de salud, es decir, no cuenta con prestaciones de trabajo que les den derecho a recibir atención médica en instituciones públicas y/o privadas; y residen 163 personas de más de 15 años que son analfabetas, de las cuales 100 son mujeres (INEGI, s.f.)

Con relación a amenazas naturales, la colonia y la ZMA están altamente expuestos a sismos, deslaves por inestabilidad de laderas y huracanes. Además, Guerrero es uno de los 13 estados en los que se encuentran el mayor número municipios considerados de alta o muy alta vulnerabilidad al cambio climático a nivel nacional (INECC, 2013). Es importante señalar que México está altamente expuesto a amenazas naturales: aproximadamente un tercio de la población y dos quintos de su territorio están expuestos a erupciones volcánicas, sismos, tormentas tropicales e inundaciones (GFDRR, 2015). Además, está altamente expuesto al cambio climático: $68.2 \%$ de la población, $15 \%$ del territorio y $71 \%$ del producto interno bruto están expuestos a efectos negativos de la crisis climática (CICC, 2009).

\section{RESULTADOS}

\section{Involucramiento y sensibilización}

El proceso de involucramiento se basó en la conformación de un grupo de mujeres y niñas de entre 5 y 72 años, el cual en su primera reunión acordó organizar un grupo de Zumba. Organizadas de lunes a viernes, las sesiones de zumba generaron alegría entre las participantes, contribuyendo a que estas se abrieran al dialogo sobre los riesgos de desastres y el cambio climático, y se implicaran en el desarrollo de la estrategia (ver Figura 2). Asimismo, desde el principio se conformó un Comité de Gestión del Riesgo de Desastres (CGRD) con la participación de las mujeres de distintas partes de la colonia para gestionar apoyos, promoviendo el mejoramiento de la colonia. 
Para comunicarse, se organizó un grupo de WhastApp llamado "Mujer y gestión del riesgo" donde se compartía no solo información sobre los riesgos de desastre y el cambio climático, sino también fotografías y videos del grupo de zumba. A través de la observación participante, desde el inicio del proceso de involucramiento se identificó la inexistencia de espacios y actividades donde las mujeres se pudieran reunir y sentirse libres de hacer cosas y sin avergonzarse de sus cuerpos.



Figura 2. Grupo de Zumba conformado por mujeres de la colonia Palma Sola FOVISSSTE Fuente: Autores, 2020

El proceso de sensibilización se realizó de manera simultánea con el involucramiento. Entre otros, se organizó una campaña durante el Día Internacional para la Reducción del Riesgo de Desastre para la cual se preparó un anuncio para perifoneo con cuatro voces con la frase "en cada momento y en cada lugar yo reduzco el riesgo de desastres", y se creó un logotipo con apoyo de un diseñador gráfico. Asimismo, se implementaron tres actividades de sensibilización. La primera fue la Cobija que tiene por objetivo esclarecer como el calentamiento global es generado por el efecto invernadero (EECO, 2016). Esta técnica permitió a las participantes tener una vivencia relacionada con el cambio climático:

"Esto es lo que está pasando con el planeta, así se siente con calor tremendo y me pone muchas veces de mal humor, lo ideal es sembrar más árboles para que esté fresco el entorno, ya no hay que quemar basura".

Participante de la actividad, comunicación personal, 26 marzo de 2018.

La segunda actividad fue el Juego de la Oca el cual busca reflexionar sobre la crisis climática (causas, efectos y acciones de mitigación y adaptación) (EECO, 2016). Entre los elementos reflexionados relacionados con la colonia destacan la quema de basura, la deforestación e incendios en la parte alta de la colonia (ver Figura 3). Esta reflexión permitió a las participantes considerar ideas para enfrentar dichos desafíos:

"sería bien que sembráramos más árboles y los cuidáramos, así como no tirar basura en la calle para que cuando llueva no se tapen las alcantarillas, también sembrar árboles frutales para hacer un huerto en casa, ya hay que cuidar el planeta".

Participante de la actividad, comunicación personal, 26 de marzo de 2018.

La tercera actividad fue el Memorama de riesgos y cambio climático el cual tiene por objetivo reflexionar sobre amenazas y peligros hidrometeorológicos, la vulnerabilidad social, la reducción del riesgo de desastres gracias a la adaptación y la organización social (EECO, 2016).

Cabe resaltar que en una primera discusión grupal, las mujeres comentaron no conocer los riesgos en la colonia. No obstante, seis meses después, se realizó una segunda discusión grupal durante la cual las participantes comenzaron a identificar situaciones de riesgo: 
"considero que, si se viene una roca, quizás y si llegue a mi casa por la velocidad, yo pensaba que quizás a mí no me afectaban las rocas porque no vivo cerca como la señora Ambrosia, pero si tengo riesgo, si las rocas se vienen llegarían entonces hasta acá abajo donde yo vivo".

Participante grupo focal, comunicación personal, 23 de agosto de 2018.

Así también señalaron que llegaron a vivir a la colonia por necesidad, ya que los sueldos son bajos y las rentas altas para vivir en lugares más seguros.

Durante las actividades de sensibilización se identificaron las siguientes barreras. Primero se identificó una dificultad para sacar a las mujeres de sus roles y actividades, principalmente ligadas al cuidado de la casa, y centrarse en temas de gestión de riesgos, cambio climático y desarrollo local. Por ello, se buscó distraerlas y permitirles realizar actividades diferentes a su cotidiano como participar en talleres, jugar y bailar zumba. Además, se consideraron sus horarios y actividades para las hijas e hijos. Segundo, se identificó una dificultad para comprender conceptos y términos debido a las condiciones de rezago educacional y en particular el analfabetismo en el cual se encuentran varias mujeres de la colonia. Más aún, ligado a lo anterior se identificó inseguridad al participar en las actividades, y una baja autoestima y la idea de que si no sabían leer o escribir como iban a aprender sobre los riesgos de desastre y el cambio climático. Frente a esto, el desarrollo de materiales y contenidos a la medida de las mujeres de Palma Sola permitió superar sus dudas con respecto a sus capacidades, mientras que al pasar el tiempo y gracias a proceso de involucramiento las mujeres ganaron más confianza y hubo mayor participación.



Figura 3. Actividades de sensibilización: Juego de la Oca del cambio climático Fuente: Autores, 2020.

\section{Diagnóstico y planeación}

Posteriormente, se realizó un diagnostico centrado en amenazas, vulnerabilidades, capacidades existentes y se elaboró un plan de acción (ver Figura 4). Con respecto a las amenazas, se identificó que la colonia esta principalmente expuesta a sismos, tormentas tropicales, inundaciones por el desbordamiento de ríos en temporada de lluvias, deslizamientos de tierra y rocas, vientos fuertes y lluvias intensas dada su ubicación (en las faldas del cerro de El Veladero) y presencia de los ríos El Camarón y Aguas blancas. Específicamente, a través de un mapeo con drones realizado con mujeres y técnicos, se obtuvo una fotografía de la situación actual que permitió corroborar la existencia de viviendas dentro o cerca de arroyos y ríos y expuestas a deslizamientos. Cabe resaltar que el riesgo asociado a fenómenos hidrometeorológicos se materializó en 1997 con el huracán Paulina, el cual afectó severamente la zona de El Zapotilllo, marcando la vida de las personas que ahí viven:

"Llevo 25 años viviendo en la calle Zapotillo, cuando ocurrió el huracán Paulina, pues yo estaba embarazada, tenía mis 2 niños, los 2 primeros grandes, si nos dormimos, estaba lloviznando cuando nos acostamos a dormir, ya nos despertamos con el ruido de la lluvia, y ya nos dimos cuenta, ya está limpio en donde mis vecinos vivían, empezamos a gritarles a 
los demás vecinos más arriba, y ya empezaron a buscar, encontraron los primeros cuerpos de los 2 niños y este y... después encontraron a una niña viva todavía y así. Yo me sentí muy triste de haber perdido a mis vecinos, vi a mis vecinos un día antes bien y ya al otro día ya no los vi, me sentía decaída, no sabía ni que hacer".

Sra. Lazara, comunicación personal, 24 de febrero de 2018.

Actualmente, Protección Civil Municipal tiene catalogada a 10 manzanas y 54 lotes de la Colonia como de alto riesgo debido a laderas altamente inestables, socavamiento, arrastre e inundación deslizamiento de rocas y talud inestable. Frente a esta situación, recomendó implementar medidas de mitigación como muros de contención y protección para estabilizar el terreno y encauzar las aguas pluviales, y en algunos casos sugirió que no fueran habitados por el alto grado de exposición. No obstante, a la fecha estas recomendaciones no se han implementado.

Con respecto a la vulnerabilidad social, de acuerdo con Consejo Nacional de Evaluación de la Política de Desarrollo Social (CONEVAL) la colonia presenta un muy alto grado de rezago social; ello implica carecías importantes en educación, salud, servicios básicos y vivienda, entre otros. Además, parte de la colonia es considerada como Zona de Atención Prioritaria (ZAP), es decir como un área "cuya población registra índices de pobreza, marginación indicativos de la existencia de marcadas insuficiencias y rezagos en el ejercicio de los derechos para el desarrollo social" (CONEVAL, s.f.b). La vulnerabilidad también está ligada a la situación de inseguridad legal de sus predios (asentamientos irregulares o en proceso de compra de los predios), baja calidad de sus viviendas (construidas en cartón, madera y mampostería débil) y carencias de servicios públicos (principalmente agua). De las 632 viviendas censadas en la colonia 514 disponen de sanitario, 503 de drenaje y 179 de aguan entubada; y tan solo 173 disponen tanto de luz eléctrica como de agua entubada y drenaje. Con respecto a acceso a internet, solo 92 casas cuentan con este servicio (INEGI, 2010). Los servicios públicos también son limitados: existe escasez de agua, y a pesar de que hay servicio de recolección de basura, hay quema de basura o acumulación de esta en terrenos baldíos; y a pesar de contar con un centro de salud este no tiene medicamentos. Además, la colonia presenta una degradación del medio ambiente: deforestación inducida por la expansión de asentamientos irregulares e incendios forestales en el parque el Veladero; y erosión por cría inadecuada de cerdos, incrementando el riesgo por deslave.

Por último, se identificó que la vulnerabilidad de las mujeres y la colonia está ligada a un patrón de gobernanza marcado por una muy limitada relación entre la población y el municipio: existe poca relación y atención por parte de los gobiernos a las necesidades de los pobladores de la colonia. En específico, en cuestión de gestión de riesgo por parte del municipio, se observó que Protección Civil Municipal realiza un trabajo en campo muy limitado: principalmente identifican los riesgos sobre los mapas y posteriormente acuden a las colonias para realizar notificaciones sin fomentar la participación comunitaria, crear lazos de confianza con los habitantes de las colonias, ni promover interacciones directas continuas (solo en temporada de lluvias) o adaptadas al perfil de las colonias. Esta situación es reconocida por los elementos de Protección Civil.

Con respecto a las capacidades, el grupo de mujeres identificó las siguientes: organización vecinal (en tiempos de crisis las familias se han apoyado entre ellas), y organización de brigadas de limpieza y brigadas médicas —en el marco de programas gubernamentales- (ver Figura 4).

Con base en esto, las mujeres acordaron el siguiente plan de acción:

1. Reforestar con árboles zonas con laderas inestables,

2. Gestionar obra pública para la mitigación de riesgos,

3. Gestionar apoyo para mejorar viviendas y servicios públicos (agua potable, alumbrado público, etc.),

4. Gestionar proyectos productivos, e

5. Implementar huertos urbanos con árboles frutales y plantas medicinales.

Asimismo, acordaron como clave el desarrollo de las siguientes capacidades para la reducción del riesgo:

- Profundizar conocimiento sobre los fenómenos hidrometeorológicos,

- Aprender primeros auxilios, y

- Aprender a elaborar productos medicinales a través de plantas naturales. 


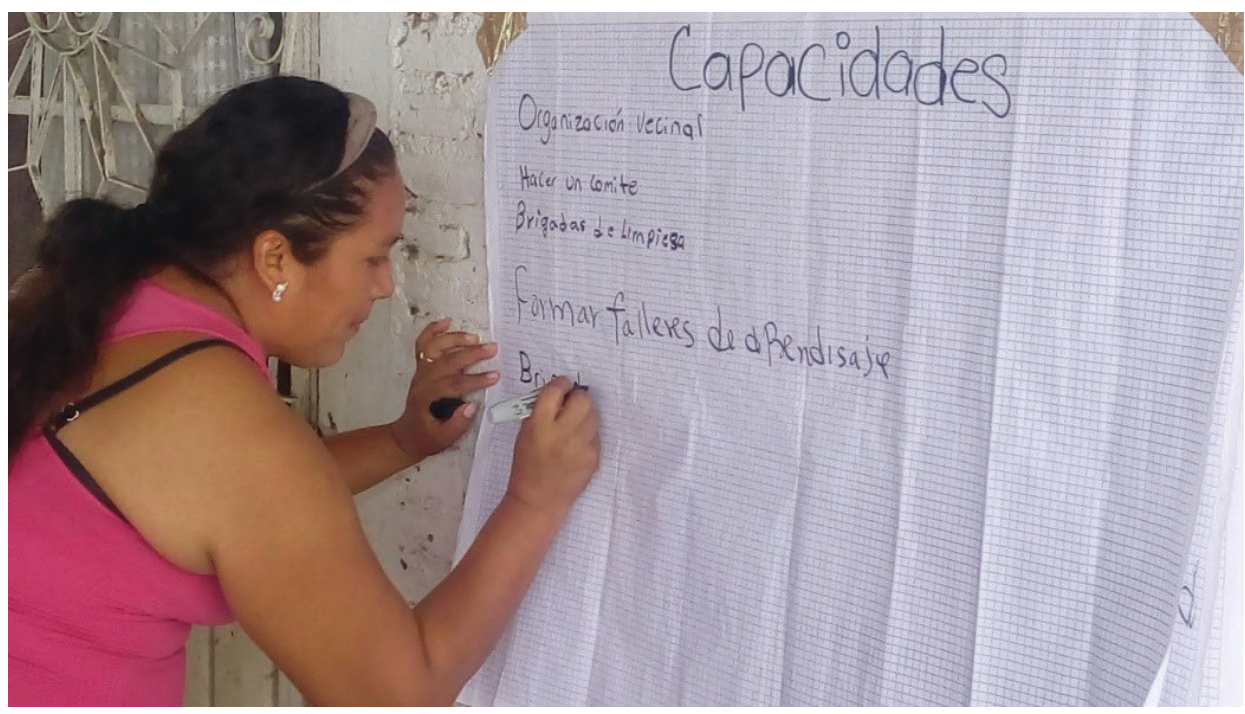

Figura 4. Mapeo de capacidades Fuente: Autores, 2020

\section{Vinculación e implementación de acciones}

El proceso de vinculación con autoridades gubernamentales implico tres grandes etapas. Primero, se realizó un mapeo de autoridades e instituciones relacionadas con la gestión del riesgo y el desarrollo local, el cual se fue consolidando de manera gradual, entre otros, gracias a los contactos que se iban generando con cada actividad de la estrategia. Segundo, para lograr el apoyo de los encargados de las direcciones o secretarias municipales para concretar las acciones del plan de acción se organizaron presentaciones en las oficinas municipales y visitas a la colonia como en la que participaron la encargada de la Secretaria Municipal del Bienestar y personal de protección civil. Cabe resaltar que el lograr que las autoridades se reunieran con el grupo de mujeres en la colonia permitió perturbar las formas de relacionarse, logrando la discusión y negociación en el espacio de las mujeres (ver Figura 5 y 6). Tercero, las mujeres acudieron a diferentes dependencias a tramitar los apoyos de manera personal ya que no se buscó apoyo político. A pesar de que los trámites para solicitar apoyos gubernamentales son personales, las autoridades permiten que actores políticos tales como los regidores gestionen por terceros. Por ello, las mujeres de Palma Sola FOVISSSTE tuvieron que tomar tiempo para hacer las gestiones en el ayuntamiento; en una ocasión las mujeres tardaron más de 2 horas haciendo un trámite.

El proceso de vinculación también incluyó acercamientos con el sector académico, sociedad civil organizada y expertos distintos temas como comunicación y botánica, los cuales acordaron apoyar el desarrollo de la estrategia de manera voluntaria. Las vinculaciones tanto con actores gubernamentales como no gubernamentales fueron facilitadas por las conexiones previas que se habían forjado en los últimos diez años trabajando en gestión y organización en otras colonias de Acapulco.

A partir de la vinculación se implementaron las siguientes acciones. Por un lado con Protección Civil Municipal se gestionaron dos talleres para el desarrollo de capacidades: uno sobre Introducción a los fenómenos hidrometeorológicos, y otro sobre primeros auxilios. Entre las participantes, hubo consenso sobre la relevancia de lo aprendido: "es bueno saber de todo esto ya que nos permite saber qué hacer ante alguna situación, por ejemplo, por atragantamiento uno sólo se desespera y no haces nada, se bloquea uno del susto" (participante capacitación primeros auxilios, comunicación personal, 12 enero de 2019). Es importante mencionar que para su organización el personal de Protección Civil solicitó específicamente que las mujeres no llevaran a niñas y niños porque estos podían interrumpir los talleres e impedir la concentración de las mujeres.

Asimismo, se gestionó un taller de elaboración de medicina alternativa para aprender a preparar jarabe para la tos. En el taller participaron 20 mujeres, y fue impartido por una tallerista especialista en botánica. Después del taller las participantes señalaron su importancia: "el aprender hacer medicina alternativa con las plantas naturales, permitirá estar preparadas ante alguna contingencia, incluso los niños, niñas y adultos mayores se enferman más de la tos cuando 
llueve mucho" (participante capacitación plantas medicinales, 26 de enero de 2019). Después del taller, las participantes mostraron intereses en solicitar otros talleres para aprender a elaborar otros productos como champú para el cabello, considerando la posibilidad de comercializarlos y así generar una fuente de ingresos.

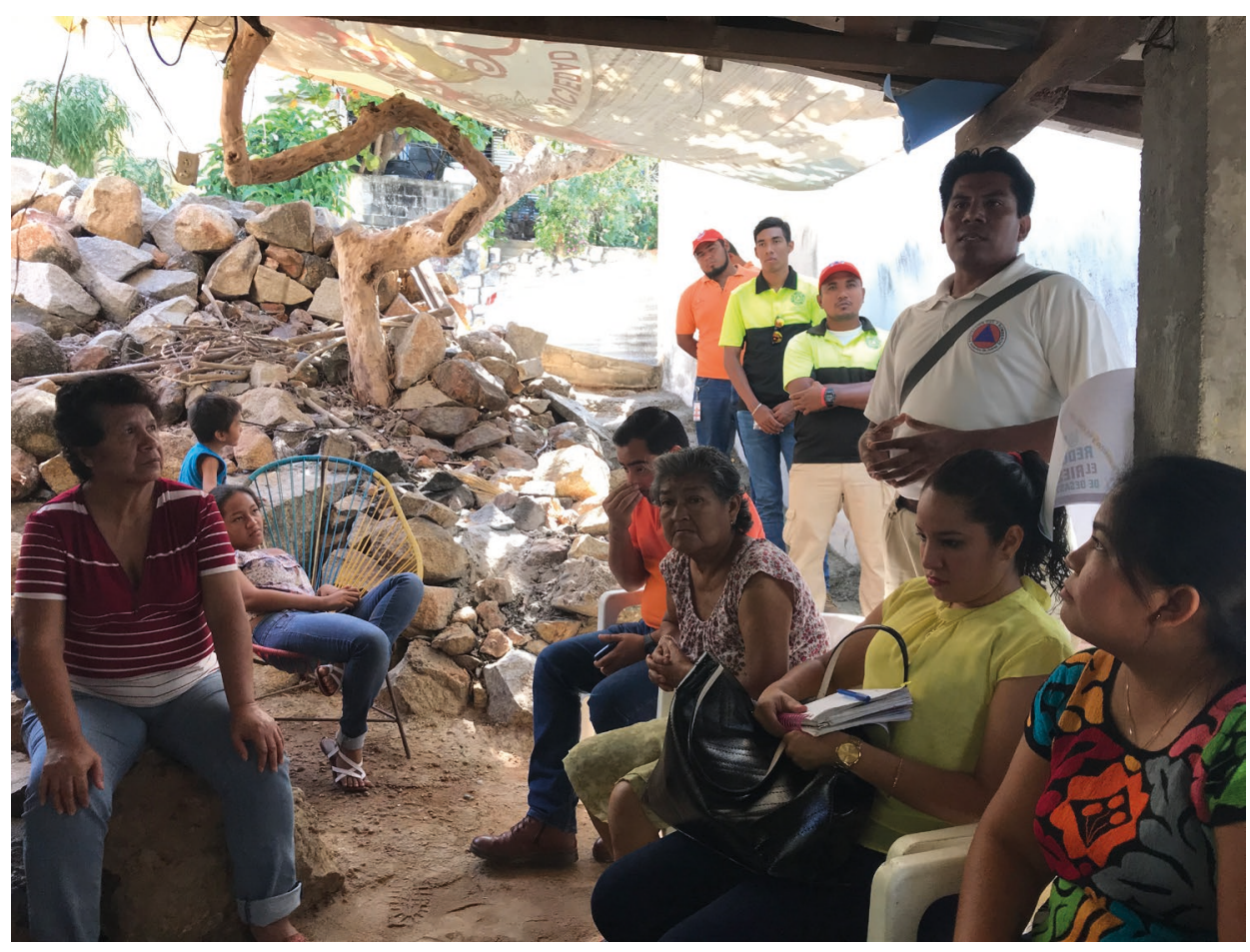

Figura 5. Visita-recorrido protección civil y secretaria del bienestar a Palma Sola Fuente: Autores, 2020.

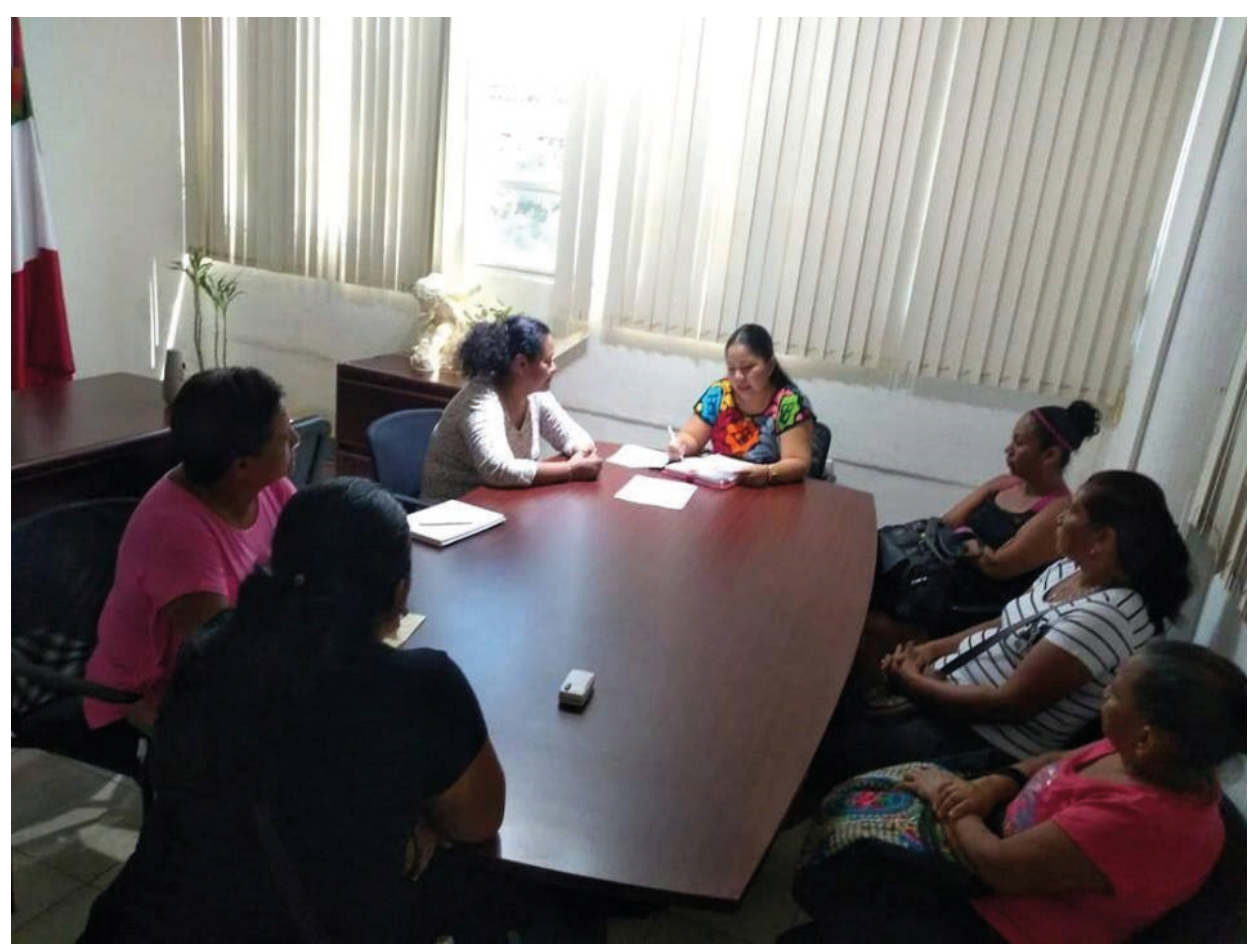

Figura 6. Reunión con secretaria del bienestar en el ayuntamiento para gestión de apoyos Fuente: Autores, 2020 
A la par de los talleres, se gestionaron mesas de trabajo con la Dirección de alumbrado público y la Comisión de Agua Potable y Alcantarillado del Municipio (CAPAMA) logrando la reparación del alumbrado público y el abastecimiento de agua potable con pipas y descuentos en multas y recargos de los recibos de agua. Por otro lado, con la Dirección General de Ecología y Protección al Medio Ambiente - Acapulco, se gestionó la donación de árboles para reforestación. Como resultado, se organizó una jornada de reforestación en la zona de Barrio Chino de la Colonia Palma Sola en la que participaron 25 personas (ver Figura 7). Se sembraron árboles frutales, enfatizando la importancia de la reforestación para reducir riesgos. Al concluir la jornada de reforestación algunas mujeres adoptaron árboles para sembrarlos en sus casas.

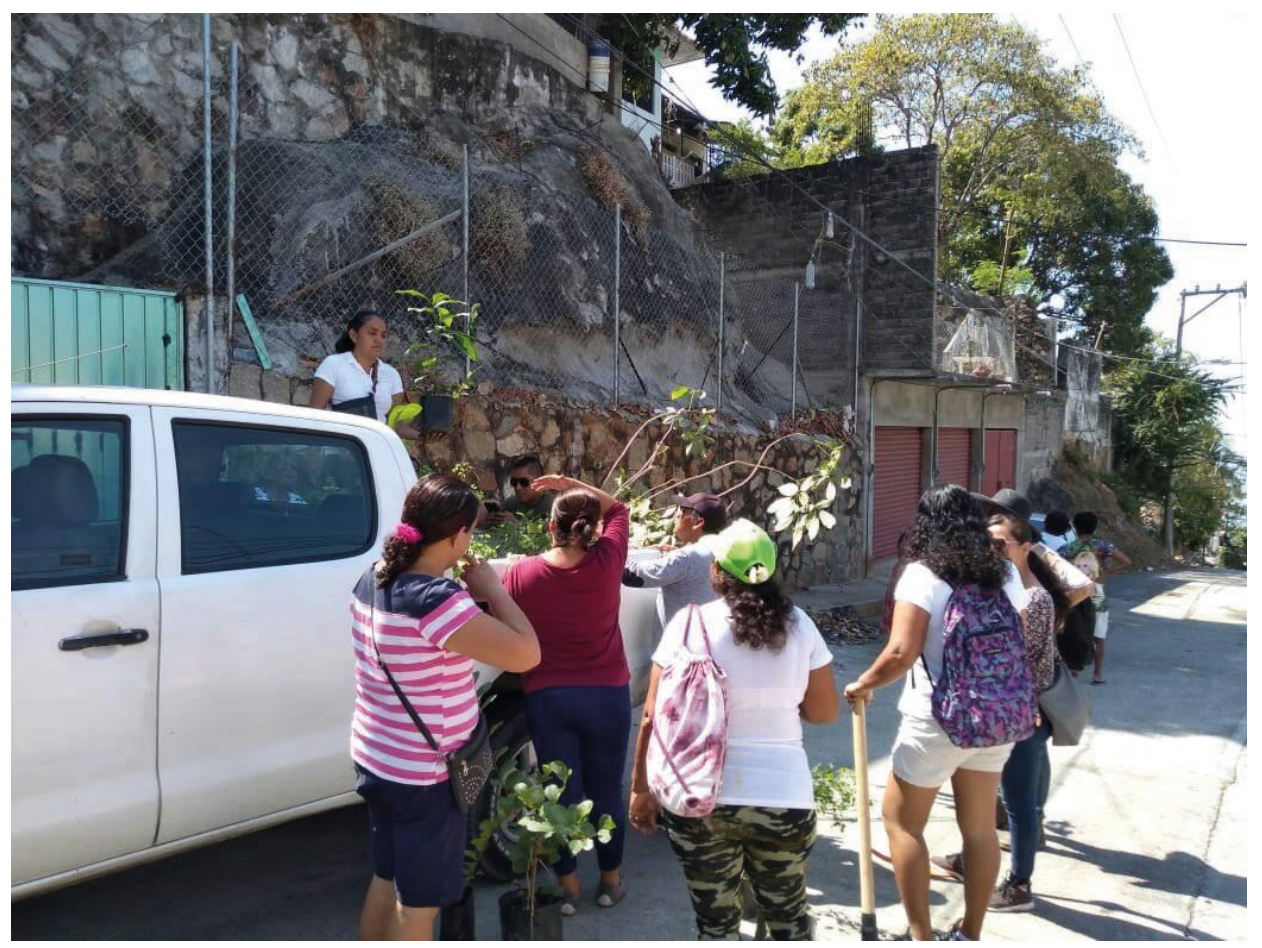

Figura 7. Jornada de reforestación Fuente: Autores, 2020

Por otro lado, se gestionó con la Dirección de Fortalecimiento Municipal un censo socioeconómico. Esto fue posible ya que parte de la colonia es considerada ZAP, dando acceso a las mujeres a participar de programas sociales y económicos (ver Figura 8). Posteriormente, las mujeres acudieron a dicha dirección para inscribirse en los programas existentes. Además, ante la Secretaría de Planeación y Desarrollo Económico Acapulco, las mujeres de la colonia gestionaron dos costureros para reducir la vulnerabilidad económica de hogares en la colonia. Las mujeres también conformaron un comité de brigadistas en colaboración con el sector salud de la colonia con el propósito de impulsar procesos de reducción de riesgos, y desarrollo de capacidades en materia de protección civil (ver Figura 9). La vinculación e implementación de acciones permitió a las mujeres involucradas ver resultados de su involucramiento en el grupo y el desarrollo de estrategia de prevención de riesgos. Esto también sirvió para llamar la atención de otras mujeres que se quisieron integrar para ver cómo podían mejorar su calidad de vida. Al finalizar el desarrollo de la estrategia, se organizó una exhibición fotográfica de las actividades realizadas en colaboración con las mujeres de la colonia, elaborando porta retratos con material reciclados. Asimismo, se creó una asociación civil para dar continuidad a lo realizado.

En torno a este eje de actuación, es importante señalar tres dificultades en los procesos de vinculación. Primero, la gestión con autoridades municipales fue dificultada porque los funcionarios desconocían temas de riesgos y cambio climático. Segundo, existe una burocracia que complica las relaciones con las autoridades gubernamental y la gestión de apoyos. Tercero, existen intereses político-electorales, que incentivan una dinámica de trueque: apoyo por voto. 


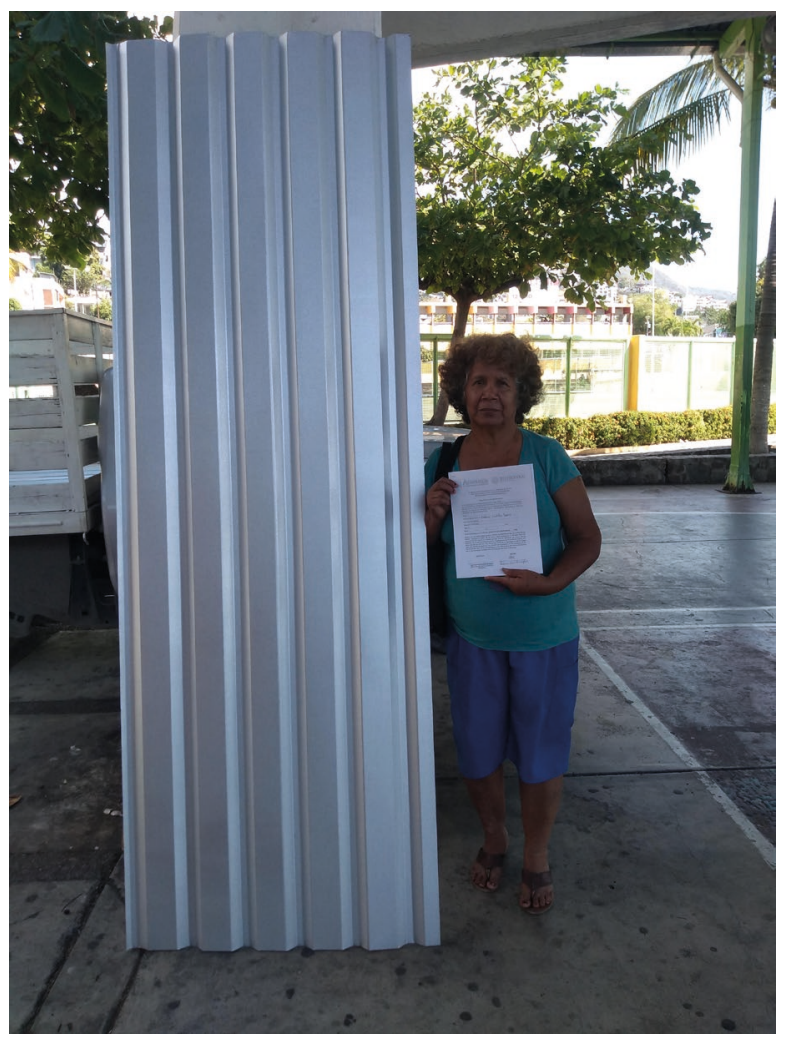

Figura 8. Recepción de materiales para mejorar vivienda Fuente: Autores, 2020

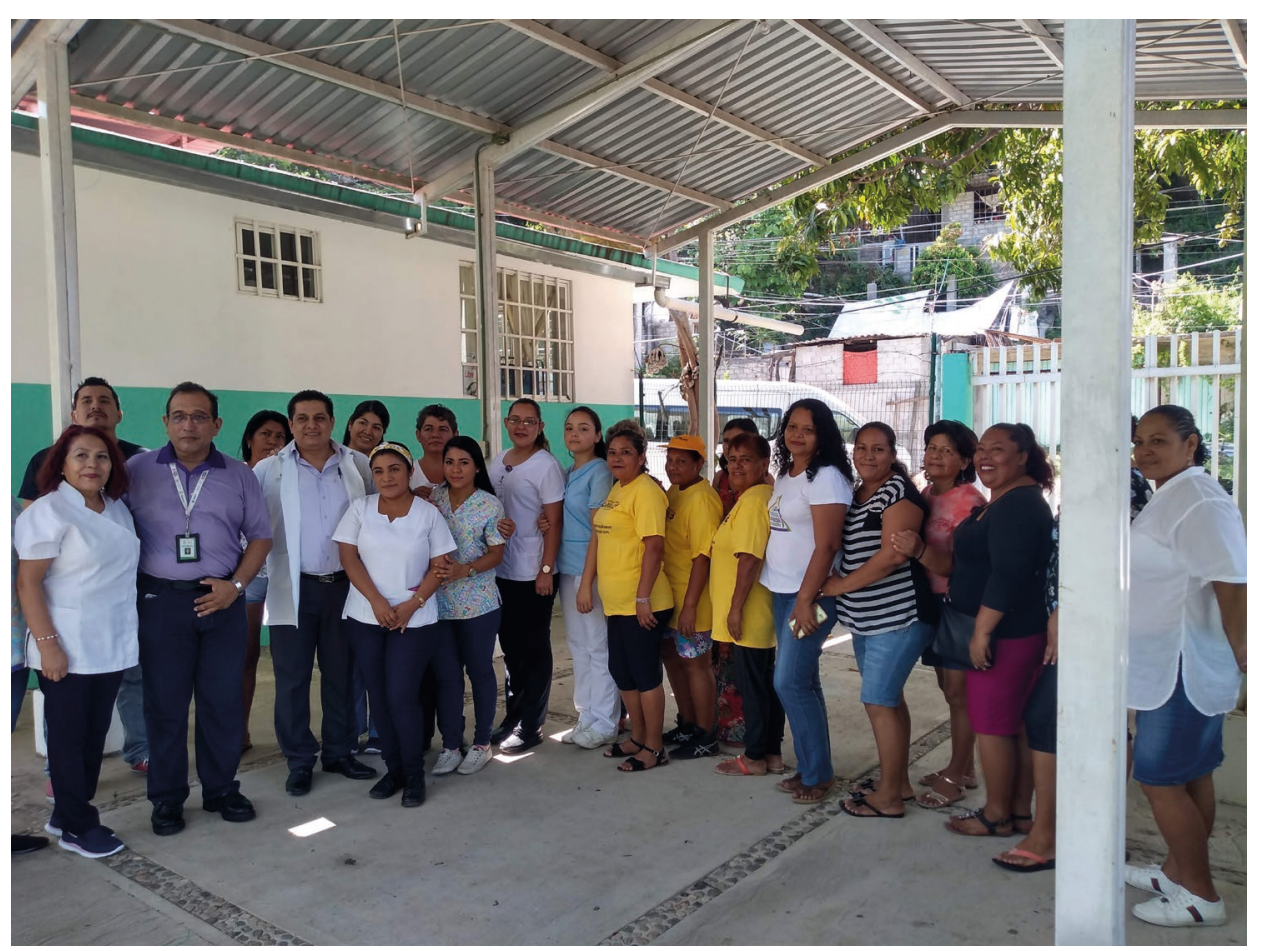

Figura 9. Conformación equipo de brigadistas (mujeres en amarillo) Fuente: Autores, 2020. 


\section{REFLEXIÓN Y APRENDIZAJE}

La estrategia diseñada e implementada en la Colonia Palma Sola FOVISSSTE nos da lecciones importantes con respecto a la puesta en marcha de una gestión del riesgo participativa y sensible al género a escala local. Por un lado, el desarrollo de la estrategia evidencio la relevancia de la implementación de los ejes de actuación tomando ventaja de las oportunidades que se iban abriendo y alianzas que se iban forjando en el tiempo. Mas aun, evidenció la importancia de los procesos de involucramiento y vinculación para sostener la estrategia. Con respecto al involucramiento del grupo de mujeres en el desarrollo de la estrategia se logró no solo gracias a las actividades de sensibilización y capacitación sobre los riesgos, sino también a través de las actividades recreativas (zumba), y la gestión de apoyos para la mejora de vivienda y del barrio (servicios). En este sentido, atender las causas subyacentes del riesgo como lo sugiere Castro (2017) no solo permite prevenir el riesgo, sino también, implicar a las mujeres en su gestión. Con respecto a la vinculación, cabe resaltar que esta implicó tanto la expansión de redes y alianzas entre el grupo de mujeres y actores gubernamentales y no gubernamentales, como el desarrollo de capacidades y conocimientos para realizar gestiones de manera autónoma.

Por otro lado, el desarrollo de la estrategia permitió corroborar la presencia de gran parte de los factores de género relacionados con la vulnerabilidad de las mujeres descritas por el PNUD (2007), incluyendo el analfabetismo, escaso acceso a tecnologías y capacitación, y la prevalencia de la división sexual del trabajo. Por ello, la gestión del riesgo con perspectiva de género es imprescindible; no obstante, su desarrollo implica grandes desafíos, dentro de los que destacan los siguientes. Primero, romper con el rol tradicional de amas de casa en el cual las mujeres se encuentran. Con base en la experiencia en la colonia, se conjetura que una parte importante de las mujeres dejaron de jugar u ocuparse de ellas desde muy temprana edad. Por ello, las actividades como los juegos y la zumba fueron clave para perturbar el cotidiano de las mujeres. Segundo, diseñar procesos y actividades a la medida de los intereses, necesidades y capacidades de las mujeres, considerando el rezago educacional para lograr una mejor comprensión sobe los riesgos y su gestión y el cambio climático sin desanimar a las participantes. Tercero, fomentar el empoderamiento de las mujeres. En el caso presentado esto se trabajó gracias a acciones para aumentar su autoestima con respecto a su apariencia física (Zumba), sus capacidades intelectuales (juegos, talleres), y sus capacidades de vinculación y gestión de apoyos con actores gubernamentales, sin intermediaros. Con respecto este último punto, es importante enfatizar que la gestión de apoyos sin intermediaros implicó para las mujeres un proceso desgastante y extenuante, que se sumó a su carga de trabajo; por lo cual emerge como importante no solo capacitar sobre cómo gestionar y desarrollar vínculos con autoridades gubernamentales, sino también simplificar procedimientos y trámites burocráticos, para evitar la desincentivación de las mujeres.

El desarrollo de la investigación en Palma Sola tuvo dos principales limitaciones: escaso involucramiento de hombres de la colonia, ligado principalmente a sus horarios de trabajo; y un acotado trabajo multidisciplinario e intersectorial, esto a pesar de la creación de alianzas que se forjaron a lo largo del proyecto. En este sentido uno de los desafíos que se vislumbran para el desarrollo de este tipo de investigaciones en otros contextos en México y la región, es la constitución de equipos multidisciplinarios y multisectoriales que permitan la complementariedad para enfrentar los problemas, trabajando tanto con mujeres como con hombres. Otro desafío es entrelazar "investigación y acción", articulando las necesidades, intereses y aspiraciones de los participantes académicos y no académicos. Un último desafío es ganar la confianza de las personas y mantener su interés a lo largo del proceso de investigación-acción, el cual tiende a ser abierto, de largo plazo y demandante en tiempo y esfuerzo para todos los involucrados.

\section{CONCLUSIÓN}

Este trabajo planteo y discutió una estrategia para incentivar la prevención del riesgo a escala local y sensible al género. Esta estrategia se basó en una investigación-acción participativa realizada con un grupo de mujeres de la Colonia Palma Sola en Acapulco de Juárez entre 2018 y 2019, y constó de tres ejes complementarios de actuación: involucramiento y sensibilización; diagnóstico y planeación; y vinculación y acción. El trabajo da cuenta del proceso de involucramiento y sensibilización basados en actividades recreativas como clases de zumba, y juegos de sensibilización. Con respecto al diagnóstico y planeación el trabajo señaló las diferentes vulnerabilidades presentes en la colonia, incluyendo mala calidad de las viviendas, carencia 
de servicios públicos y escasa vinculación con autoridades gubernamentales. Por último, en la vinculación e implementación, el trabajo ahondó en el desarrollo de relaciones con autoridades gubernamentales y no gubernamentales y la implementación del plan de acción.

Partiendo del consenso sobre la importancia de centrar la gestión del riesgo en acciones de prevención y reducción del riesgo, esto desde un enfoque participativo y de género, este trabajo contribuye a reflexionar sobre las formas de concretar dicha perspectiva a escala local. Especificamente, emergen dos lecciones importantes sobre el desarrollo de una de gestión del riesgo participativa y sensible al género que disminuyan la vulnerabilidad social e incrimine las capacidades. Por un lado, este trabajo enfatiza la importancia de generar estrategias sostenidas por procesos involucramiento que busquen entrelazar la gestión del riesgo y el desarrollo local, interesando e implicando a las mujeres en materia de riesgos y cambio climático; y procesos de vinculación que permitan a las mujeres tejer alianzas con actores gubernamentales y no gubernamentales y desarrollar capacidades de gestión y organización, fomentando procesos de empoderamiento. Por el otro, corroborando la persistencia de los factores de genero ligados a la vulnerabilidad de las mujeres, este trabajó subraya tres desafíos para desarrollar una gestión del riesgo con enfoque de género: romper con los estereotipos y roles de género, diseñar procesos y actividades a la medida de los intereses, necesidades y capacidades de las mujeres, y fomentar el empoderamiento de las mujeres, buscado en particular mejorar su autoestima y expandir sus capacidades para relacionarse y crear alianzas multisectoriales.

Dos principales líneas de investigación y de acción surgen a partir de este trabajo. Primero, cómo mantener los procesos a largo plazo; específicamente, qué alianzas, qué financiamientos y métodos de intervención pueden ser diseñando implementados para consolidar lo iniciado e ir adaptándolo y adoptándolo en otras colonias de Acapulco. Segundo, cómo lograr articular este tipo de estrategias locales con proyectos e intervenciones sobre reducción de riesgos, desarrollo local y acción climática en Acapulco y su zona metropolitana; ello, sin perder el involucramiento e interés de las mujeres, considerando las barreas educativas existentes y los estereotipos y roles de género, los cuales tienden a acotan su participación.

En las últimas décadas se ha llegado a un consenso sobre la relevancia de desarrollar estrategias de prevención del riesgo a escala local y sensibles al género. Hoy en día es esencial continuar avanzando, explorando formas de concretar dicho consenso a fin de transitar hacia un desarrollo resiliente, sostenible e inclusivo en la región.

\section{AGRADECIMIENTOS}

Se agradece infinitamente el apoyo al grupo de mujeres de la Col. Palma Sola FOVISSSTE, especialmente a la Sra. Ester Bernabé Plaza, quien desde un inicio apoyó en todas las acciones del proyecto de investigación-acción participativa, y es quien a la vez motivó al grupo para la gestión de apoyos individuales y colectivos con el fin de mejorar la calidad de la vida de las mujeres y hombres de la colonia.

\section{REFERENCIAS}

Becerril, H., Rodríguez, L., \& Yáñez, K. (2020). Patrones de gobernanza posdesastre en ciudades intermedias: aprendizajes desde la Zona Metropolitana de Acapulco. Artículo en preparación.

Brydon-Miller, M., Greenwood, D., \& Maguire, P. (2003). Why Action Research? Action Research, 1(1), 9-28. https://doi.org/10.1177/14767503030011002

Aguilar, L., Castañeda, I., \& Salazar, H. (200o). En búsqueda del género perdido: equidad en áreas protegidas. UICN - Absoluto.

Arana, M. T. (2017). Caso de estudio: género y cambio climático en América Latina. Alianza Clima y Desarrollo. Recuperado de https://cdkn.org/resource/generolac/?loclang=es es

Boyano, D. (2013). Género y disminución de riesgos de desastre en Ecuador. Secretaria Nacional de Gestión de Riesgos (SNGR) \& Instituto Nacional Geológico Minero Metalúrgico del Ecuador (INIGEMM). Recuperado de http://generourban.blogspot.com/2015/o7/genero-y-disminucion-deriesgo-de.html

Castro, C.P. (2017). Reder, una revista para avanzar en la reducción del riesgo de desastres y el mejoramiento del bienestar humano. Revista de Estudios Latinoamericanos sobre Reducción del Riesgo de Desastres REDER, 1(1), 1-5. http://www.revistareder.com/ojs/index.php/reder/article/ view/1 
Comisión Económica para América Latina y el Caribe CEPAL. (2018). La economía del cambio climático en américa latina y el caribe. Una visión gráfica. CEPAL. Recuperado de https://www.cepal.org/es/ publicaciones/42228-la-economia-cambio-climatico-america-latina-caribe-vision-grafica

Comisión Intersecretarial de Cambio Climático CICC. (2009). Programa especial de cambio climático 2009-2012. Gobierno de México. Recuperado de http://www.semarnat.gob.mx/archivosanteriores/ programas/Documents/PECC DOF.pdf

Colmenares, A. (2012). Investigación-acción participativa: una metodología integradora del conocimiento y la acción. Voces y Silencios: Revista Latinoamericana de Educación, 3(1), 102-115. https://dialnet.unirioja.es/servlet/articulo? codigo $=4054232$

Consejo Nacional de Evaluación de la Política de Desarrollo Social CONEVAL. (s.f.a). InfoPobreza. CONEVAL. Recuperado de http://sistemas.coneval.org.mx/InfoPobreza/

Consejo Nacional de Evaluación de la Política de Desarrollo Social CONEVAL. (s.f.b). Medición de la pobreza. CONEVAL. Recuperado de https://www.coneval.org.mx/Medicion/Paginas/PobrezaInicio. aspx

Espacio de Encuentro de las Culturas Originarias EECO. (2016). Metodología. Proceso participativo con enfoque de género e intercultural para atender el cambio climático a nivel local. EECO. Recuperado de https://eeco.org.mx/wp-content/uploads/2018/07/Metodolog\%C3\%ADa.pdf

Global Facility for Disaster Reduction and Recovery GFDRR. (2015). Country profile: Mexico. GFDRR. Recuperado de https://www.gfdrr.org/en/publication/country-profile-mexico

Instituto Nacional de Ecología y Cambio Climático INECC. (2013). Vulnerabilidad al cambio climático en los municipios de México. INECC. Recuperado de http://peacc.jalisco.gob.mx/ BoletinMunicipiosVulnerablesalCambioClimaticoINECC.pdf

Instituto Nacional de Estadística y Geografía INEGI. (s.f.). Censo de Población y vivienda 2010. INEGI. Recuperado de https://www.inegi.org.mx/sistemas/olap/proyectos/bd/censos/cpv2o10/ pt.asp?s=est \&c $=27770 \&$ proy $=$ cpvio $\mathrm{pt}$

INEGI. (2016). Panorama sociodemográfico de Guerrero 2015. INEGI. Recuperado de http://internet. contenidos.inegi.org.mx/contenidos/Productos/prod_serv/contenidos/espanol/bvinegi/productos/ nueva_estruc/inter censal/panorama/702825082208.pdf?platform=hootsuite

Intergovernmental Panel on Climate Change IPCC. (2018). Global warming of $1.5^{\circ} \mathrm{C}$. IPCC. Recuperado de https://www.ipcc.ch/sri5/

Lavell, A. (2008). Una visión de futuro: La gestión del riesgo. PAHO.

Oficina de Naciones Unidas para la Coordinación de Asuntos Humanitarios OCHA. (2020). Desastres naturales en América Latina y El Caribe 2000-2019. OCHA. Recuperado de https://reliefweb.int/ report/world/desastres-naturales-en-am-rica-latina-y-el-caribe-2000-2019

Programa de las Naciones Unidas para el Desarrollo PNUD. (2007). Superar la desigualdad, reducir el riesgo. Gestión del riesgo de desastres con equidad de género. PNUD.

Secretaria Bienestar. (s.f.). Sistema de Información Social y Georreferenciada. Gobierno de México.

UN Office for Disaster Risk Reduction UNDRR. (2015a). Disaster Risk - Disaster risk reduction E disaster risk management. UNDRR. Recuperado de https://www.preventionweb.net/risk/drr-drm

UNDRR. (2015b). Marco de Sendai para la Reducción del Riesgo de Desastres 2015-2030. UNDRR.

UNDRR. (2019). Global assessment report on disaster risk reduction 2019. UNDRR. Recuperado de https://gar.undrr.org/report-2019

Wilches-Chaux, G. (1998). Auge, Caída y Levantada de Felipe Pinillo, Mecánico y Soldador o Yo voy a correr el riesgo. Red de Estudios Sociales en Prevención de Desastres en América Latina. Recuperado de https://www.desenredando.org/public/libros/1998/gglr/GGLR todo ene-7-2003.pdf

Zhenmin, L., \& Espinosa, P. (2019). Tackling climate change to accelerate sustainable development. Nature Climate Change, 9(7), 494-496. https://doi.org/10.1038/s41558-019-0519-4 